\title{
Sustainable procedures for environmental evaluation of building materials and technologies
}

\author{
M. C. Forlani, M. M. Lepore \& A. Basti \\ Department of Technologies for Built Environment, \\ University “G. d'Annunzio”, Chieti-Pescara, Italy
}

\begin{abstract}
The instances of sustainability in building construction have produced some particularly interesting research both at the level of "production" of architecture and at the level of "evaluation" of the technical solutions proposed. Sensitivity towards environmental problems seems to have reached the conscience of users and those working in the building sector; however it is proving hard to achieve an effective new approach to planning. But how can this new conscience be "built up"?

The notes that follow are the synthesis of a study that is underway concerning an approach to planning that is aware of environmental criticality and indications to make more sustainable choices. The test is applied to a case study of the wealth of mud and earth buildings in the Abruzzo region.

In particular reflections are made:

a. to set out a planning method that moves from a careful analysis of the possibilities offered by the environment, in terms of a stratified building culture and awareness of physical phenomena. A reading of tradition provides a wide repertory of solutions that should in any case be carefully evaluated, adapted and developed, in a word, put into practice to then adapt it to contemporary needs, making the best use of the knowledge of the technology of materials, the physical technique, of physiology.

b. to hint at the instruments to achieve the aim of a sustainable development (economic and social) - though self construction (limited only to cases of rebuilding or restoration) or industrial production - that takes into account the environmental questions.

c. to lay out a test that can be applied in a minimum module, emblematic of the paradigm of the technical and environmental appropriateness of a dwelling system in relation to a determined physical-climatic context.
\end{abstract}

Keywords: raw earth, eco building, life cycle assessment. 


\section{The case study}

In the case of this study, we tried to move from the indications observed in order to realize sustainable interventions, connoted also by a morphological quality, by using the preexistences in raw hearth on the regional territory. However, different problems subsist for the building $\mathrm{s}$ in raw earth of the rural territory of Abruzzo region. The prejudice and the distrust towards the use of the material "raw earth" has ancient and well known roots, connected to a difficult socialeconomic past and to a cultural climate that is slightly opened to selfconstruction practices.

To give dignity to such a patrimony again, one tried to trace illustrious similarities/analogies in the works of "Masters": together with the treatment of the local product some plans by Fathy and LeCorbusier have been investigated; in fact, the first part of the work moves side by side from a particular reading of the constructions in raw earth, that can be reasonably considered as emblematic of a typical Mediterranean, sustainable and ecological heritage, from which it is possible to recognize a dimensional- materic "module" that identifies a "system" tightly interconnected to a structural, environmental climatic and functional level: in the specific instance:

- $\quad$ from Hassan Fathy we have achieved a three-dimensional module, due to technical-structural and physical -environmental needs;

- from LeCorbusier a "family hearth" comes out, that has to be realized according to the fundament al demands of the user.
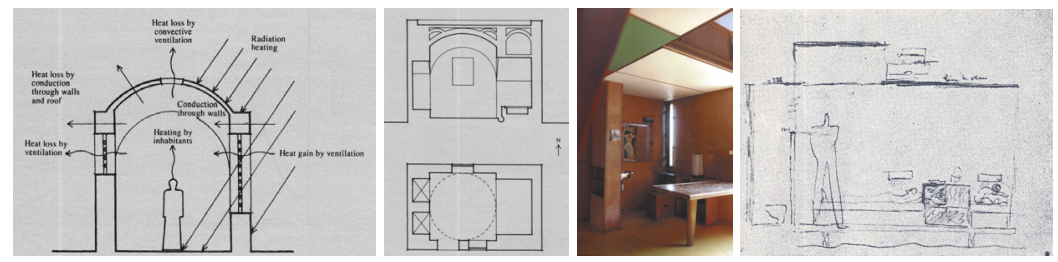

Figure 1: The three-dimensional module of H. Fathy and The "Cabanon" of Le Corbusier.

The analogous "module", found at the base of the bare, local living system and in the research of the "masters", allowed the tracing of a methodology to propose to the technicians and to the Administrations useful suggestions and to pursue, with appropriateness, the recovery and the planning of new manufactured articles in particularly sensitive and vulnerable areas (Forlani [1]).

In this situation the "project" has to become, more than in other cases, "careful" and appropriate to the context, which is to be intended in a wide sense: the sustainable design of habitations that have to reduce the consumptions and the wastes to the minimum in order to get high-level performances and comfort. Moreover, we sought to experiment the interventions in order to reconstruct continuity with a local Mediterranean identity. 


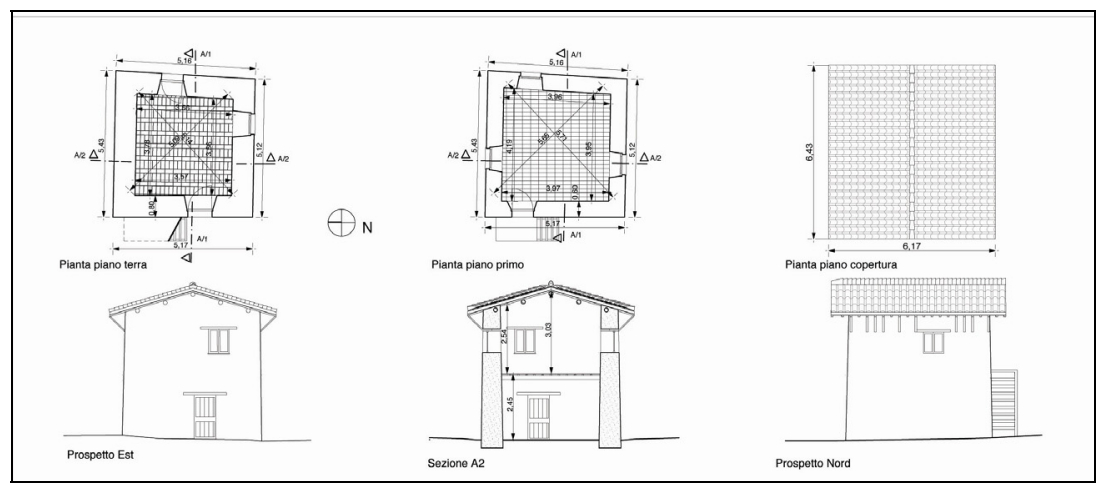

Figure 2: $\quad$ A relief of local building (case study).

Therefore, the methodology takes upon itself the task of realizing the usual demands to be born in mind in the "plan" in the light of environmental issues, that is, the use of resources, polluting emissions, the production of waste products and utilization/protection of cultural diversities. Such criticalities, by identifying new demanding classes aimed at containing the use of resources, reducing environmental loads, at the use of local climatic resources, integration with the environmental context, in fact enrich the already known requirements of "preservation of the environment", "wellbeing" and "appearance". The parts which follow report the synthesis of the current studies with particular regard to consideration:

- of the utilization of local climatic resources (integrating the demand for "wellbeing"): the solar factor, the orientation of the manufactured article, in short the attention to the carrying out of the whole envelope, in relation to the exposure in order to minimize the external energetic supply (through the planning of the frontages, as of the cover, with a specific attention to the definition of the wall and to the distribution of the openings for the control of the heat, of the ventilation, of the illumination and of the shading). One wants to consider in particular the element of closing which can become larger, in dimensions and functions, to the point of shaping new spaces as well as contributing to furnish a consistent provision of winter comfort; this is the case in which a wall can be replaced by an attached greenhouse! In this way, one also comes to consider the demand for usability in that one meets the necessity, often felt, for further living space which thus performs the double function of spatiality and microclimatic functionality;

- of containing the use of resources (integrating the demand for "preservation of the environment") by means of the use of recyclable and local materials, such as raw earth, as well as with to start shaping the parameters of control of this conscious design, starting from an environmental evaluation and certification. 

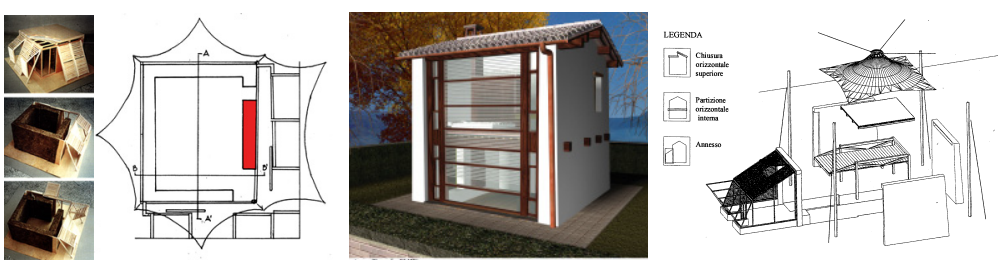

Figure 3: Three examples of evolution of the wall (by students' works).

Therefore, the aims move from the will to recover the huge present patrimony and, even more, to propose new constructions -perhaps the most appropriate in vulnerable areas a s the rural ones and mostly, for instance, for the protected territories (park areas). For these reasons, became necessary and urgent to make an afterthought of the whole building process, to adjust to the principles of the sustain ability, starting from the evaluation of the eco-industrial strategies for the reintroduction of these constructive systems in the current practice, up to the conclusion hypothesis of the manufactured article's life cycle, with the deconstruction of this latter. The suggested methodology and instruments become, from this point of view, "elements" to be further investigated for future developments, at a theoretical and applicative level.
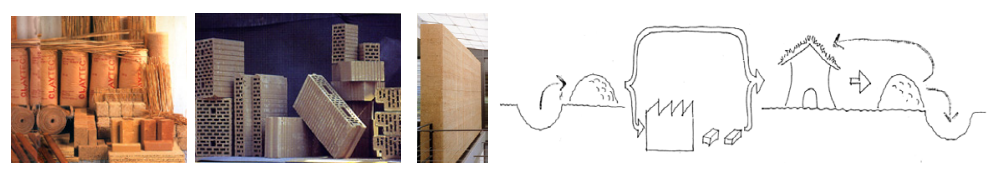

Figure 4: The components' production in raw earth and draw of their life cycle.

\section{The evaluation of the passive systems (attached greenhouse) in the comfort determination (**)}

In the light of what has been stated above, it is evident as the matching of a passive solar system with the characteristics of the "solar attached greenhouse", with a construction in raw earth is particularly appropriate. The structures with high thickness of the construction in raw earth furnish the necessary inertia for the good working of the system. Meanwhile the "lightness" and the transparencies proper of the greenhouse allow an appropriate captation of the solar energy in winter and a system of screening of the masses in the summer. When we speak about "attached greenhouses", "greenhouses or solar porches" applied to the buildings, we refer to "tampon-spaces", which are realized separating parts of the envelope from the external environment through transparent surfaces. These components have a double thermal function: the first one is that they reduce the loss of heat through the wall to which they are attached; the second one is that they carry out a function of captation of sun radiations. 
In the attached greenhouses, the wall of the room usually shows openings and transparent surfaces, that allow the internal space to earn heat, both directly and indirectly. In the daily hours of the cold seasons, the sun radiation that penetrates through the transparent surface of the greenhouse affects, in part, the floor of this latter and the partition, and partly penetrates in the controlled housing space through the openings (doors) and transparent surfaces. The part absorbed by the floor is accumulated in its mass, therefore it heats the air in the greenhouse for convection. Also the partition carries out the same function and if it is not isolated, it constitutes an accumulation that is directly in contact with the internal environment. The part of radiation that directly penetrates in the internal space, constitutes instead a direct solar benefit. The warm air of the greenhouse can penetrate in the internal space in a natural or forced way; this recalls an equivalent carrying capacity of air from the internal space toward the greenhouse. In the hours when the temperature of the air in the greenhouse is inferior to that inside (during the nocturnal hours or when there is scarce isolation), this mechanism must be inhibited to prevent from cooling the air of the place instead of heating it.

\subsection{The extension of the housing space}

We can identify different uses: temporary, permanent or sporadic use. The various utilizations indicated can be distinguished for duration and frequency. They noticed that despite the levels of variable temperature, depending on the climate, and despite the function of the greenhouse of connection between outside and inside (passage/entry), the greenhouse is used firstly as temporary living room, granting all the respective functions of it. The greenhouse puts into discussion the organization of the internal space and invites to modify it in function of the day-night and winter-summer variations. As a matter of fact, the greenhouse is like a "climatic mediator" that naturally produces a microclimate in function of the external climatic conditions.

\subsection{The greenhouse, variable element with the sun}

The envelope of a building is like the skin: it constantly reacts to the thermal variations of the external environment. A modification of the skin modifies the thermal exchange. It is necessary to give dynamism to the envelope, and to consider that this is the place where information between the inside and the external climatic conditions is exchanged. Between day and night, winter and summer, the architecture must adapt itself to the change, that is, it must be thought by keeping in mind the annual and daily cycle. This also means that living in a greenhouse, as it has been mentioned above, involves an awareness, on the part of the consumer, of these cycles and the heat transfer from one environment to the other.

It is about isolating the volume to heat during the night, and to make it permeable in the daytime with the purpose of optimizing the thermal balance during the twenty-four hours. The movable partitions, that have the function to delimit the habitable space at night, will be therefore integrant part of the project. 
Also the transformations summer-winter are fundamental to assure the comfort during the whole year. During the summer it is priority to protect the greenhouse from the sun radiancy, that is direct and diffused. In fact, in this season it is not enough to screen the greenhouse from the direct radiancy alone, since due to the conditions of the sky, that is often clear, the radiation reflected by the surrounding environment (albedo) causes a strong diffused radiancy. It is therefore necessary to anticipate however a system of evacuation of the warm air in excess, unless the whole system is made superfluous by the complete disassembly of the greenhouse, that is not always compatible with its real use.

\subsection{The thermal accumulation}

Because of the different distribution of the solar energy availability during the twenty-four hours and of the demand of heat, it is important to be able to accumulate energy that is available in the daytime to be able to use it also at night. This accumulation would allow, besides, to dampen the peaks during the period of excess availability compared to the heat demand (thermal inertia). There are different ways to realize the accumulation, that however is a fundamental element of the general conception of the greenhouse, from which depends not only the general effectiveness of the system but also the level of obtainable comfort. The greenhouse, for its nature, is suitable to be associated with passive accumulation systems, whose functioning depends both from the choice of the material utilized as accumulator, and from its position and its sizing.

Opportunely managed, the thermal mass inside the greenhouse can also contribute to the cooling of the system. In fact, if the passage of the air is allowed on the thermal mass of the greenhouse with proper openings in the fresh summer nights, it will release part of the accumulated heat. If such mass is screened by the solar rays during the day, part of the heat produced in the greenhouse will be absorbed and it will not raise the temperature of the air, with an advantage of the same greenhouse and of the adjacent environments. The heat absorbed by the thermal mass will be dissipated during the night, thus completing in this way the daily cycle. The openings positioned between the greenhouse and the adjacent environments will allow the improvement of the cooling effect, by involving these environments in the circulation of the nocturnal air.

\subsection{The thermal inertia}

The principle of the thermal inertia of a wall is manifested when a flux of energy strikes its side and this is accumulated and returned also by the other with a certain delay. This phase displacement in time is also accompanied by a reduction of the amplitude of the thermal wave that means an amortisation of the same wave. An effect definable as "thermal flywheel" is fundamental both for the comfort conditions of the same greenhouse and of the adjacent space. It must be specified that the reduction of the amplitude of the wave, does not mean a reduction of the flux. The thermal effectiveness of the inertia depends on the 
total thermal capacity, that must be sufficient to absorb and to accumulate the whole available heat, and on the extension of the exchange surface, particularly of that exposed to the solar radiancy.

An inertia to which doesn't correspond a sufficient exchange surface in relation to the relative mass, can never furnish suitable performances. In a greenhouse with a great deal of glass door surface, properly isolated but without any thermal inertia, great temperature oscillations will occur. Meanwhile, the fundamental principle that characterizes a bioclimatic structure in relation to a traditional structure is to use a sufficient thermal inertia that allows the nocturnal recovery of the heat in excess during the day. In the solar greenhouses the necessary thermal mass is constituted by the walls and by the floor.

As a last requirement, not certainly because of its importance, a correct management of the greenhouse must be assured, which is to be considered like a machine. The inhabitants must understand its functioning and know how to modify its set-up according to the external, environmental conditions.

All attached sunspaces characteristics -its lightness, the capacity of assuming different configurations, in opening and closing itself by the passage of the air, in catching and send back the sunlight, in being totally transparent or filteringmake it particularly interesting if integrated with massive building, such as those made with raw earth, where the dynamic conception of the architectural wrapper as climatic go-between is effectively applicable also for the constructive traditional techniques re-examination.

\section{The environmental behavior of materials and constructive elements $(* * *)$}

In support of an efficient integration of the aims of preservation of the environment in the process of the choice of materials and configuration of the technical elements of the building, in recent years several procedural and normative instruments of a national and international level have followed one another. Instruments prevalently aimed at the adoption of pro-active and voluntary approaches oriented towards the assessment of the whole life cycle of the products used (LCA-Life Cycle Assessment-ISO 14040,1997).

\subsection{The body of legislation}

In the case of the industrial products, the body of legislation turns out to be centred on politics of European level to promote the diffusion between the different territorial actors (local Administrations, Designers, Producers, Builders and Consumers) of environment oriented behaviors. Their contents and aims are expressed in the Green Paper on IPP (Integrated Product Policy) and are found to be realizable by means of the adoption of criteria for ecological planning (DfEDesign for Environment-ISO/TR 14062, 2002) and of procedures for the environmental labelling of the products (ecolabel-ISO 14021 and EPDenvironmental product declaration-ISO/CD2 14025, 2004). Building products are no exception. Both the DfE criteria and the LCA methodologies can be 
applied to these also for the quantification of the use of resources utilized and of the emissions released into the environment, in order to obtain their environmental certification based on the EPD procedures (ISO/DIS 21930, 2005), at this moment limited to the life cycle phases ranging from cradle to gate.

As far as the configuration of the building elements is concerned instead, the normative picture presents a more recent evolution, prevalently based on the setting up of Protocols and Guidelines for ecologically sustainable planning (Annex 31 [2]). It's about instruments aimed at integrating requirements and performance, individualized for the System-building by the Italian norms UNI 7867, 8289 and 8290, with further indicators, related mainly to the exigency class of the Environmental Protection. These Protocols, based on criterions of evaluation of qualitative and quantitative type, according to the categories of damage investigated, have the tendency to make an increasing reference to necessity of sustaining design choices by drawing up specific, detailed LCA, which take into due account all the phases of the life cycle of the technical elements (ISO/TS 21931-1, 2006).

One refers in particular to the phases at the source of the productive process, like the quality and quantity of raw materials used (and their possible reduction) and the service life of the technical elements (and its possible extension). Furthermore, one refers to the phases following the building process, corresponding to the management of the elements (maintenance and/or substitution) and the end of their life (ceasing mode, re-use and recycling of parts and materials) (Fernandez [3]). Phases not presently included within the system's limits by the EPD procedures.

\subsection{The assessment of the environmental behaviors}

The application of the methodologies and instruments just mentioned to the specific case was carried out by trying to integrate in one unique decisional process the criteria supplied by the instruments of eco-design and the results deriving from the use of assessment methodologies and instruments of environmental impact based on the analysis of the life cycle. The enquiry carried out on the building analysed, a structure in raw earth representative of the Abruzzo building tradition, investigates the environmental impact produced by single technical elements, prevalently carried out with local materials and realization techniques. In this specific case, the assessment analyses concerned the building elements mainly representative of the study subject, in terms of their role (sustaining structure, external closure) and of the quantity of materials used. It concerns the masonry (56\% of the total in weight), the covering and the greenhouse leaning against it. Furthermore, for some of these, alternative building solutions were hypothesized, so as to compare the results (Basti [4]).

The environmental assessment has been performed using calculation methods already experimented in the European range (Echo-indicator 99-Holland, EPS 2000-Sweden) and employing international data bank for the knowledge of the specific impact models that are connected to the single materials and processes 
(Hendriks [5]). Some processes, in particular those related to the raw earth, have been implemented ex novo during the job.

Because of limited space, only the results concerning the supporting wall have been reported, which has been analysed with reference to two building hypotheses: in raw earth ("massone" technique) and in solid brick. It must be stressed that, in the analysis, the phase of management of the technical elements (maintenance and restoration of the parts) was not calculated in that it emerges from literature that, for technologies of a low complexity level such as those reference was made to, it presents an incidence percentage that is quite contained (about $2 \%$ of the total of the impact generated along the entire life cycle of the technical element). The graphs refer to the environmental results elaborated according to the eco-indicator 99 method, egalitarian version. To be noted is the different reference used in the building phase to the supplying of the "earth" material (found and transformed on the site) and to the production of bricks.

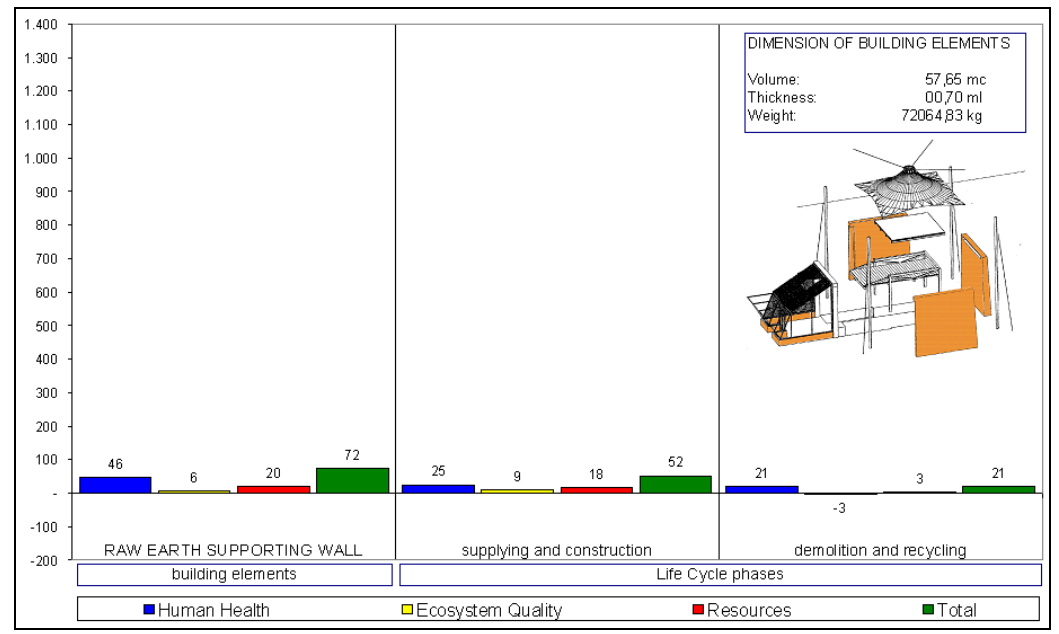

Figure 5: Environmental outcome of the supporting wall in raw earth, Analysis by categories of damage. Eco-indicator 99 method, egalitarian version.

The result we obtained by our work of research allows us to make some considerations. On the same level as the typology of the realized processes used (mainly of industrial character) the recourse to natural materials, locally available, is inclined to take shape as one of the strategies with the smallest environmental impact. The same result is extended to the production of the constructive elements, whereas they are obtained appealing to appropriate technologies, high-intensities of work and low-energetic content. 


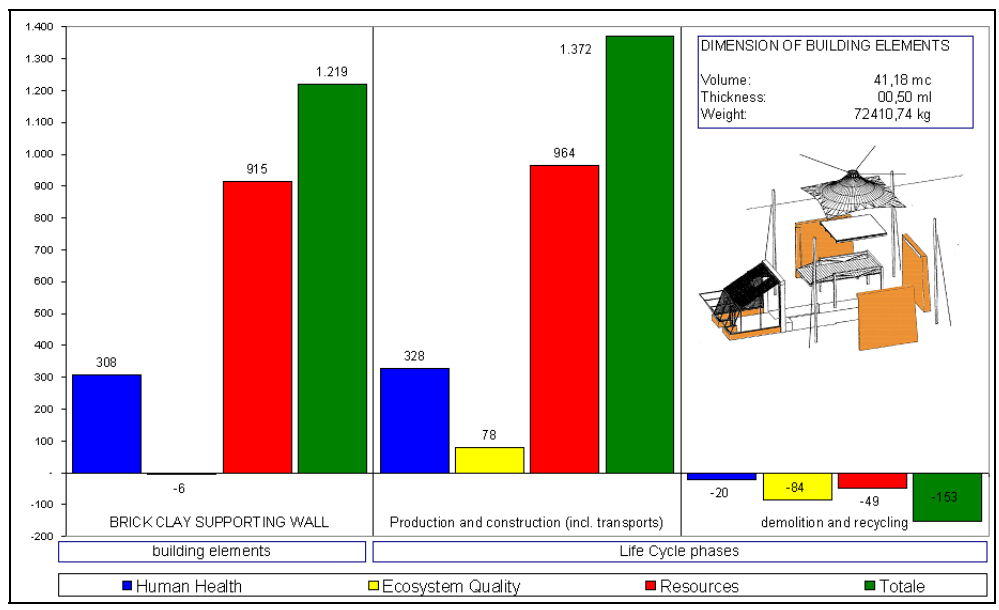

Figure 6: Environmental outcome of the supporting wall in clay brick, Analysis by categories of damage. Eco-indicator 99 method, egalitarian version.

\section{References}

[1] Forlani M.C., Costruzione e uso della terra, Maggioli editore, Rimini, 2001

[2] Annex 31, Energy-Related Environmental Impact of Buildings. Comparative applications: a comparison of different tool, CMHC on behalf of IEAECBCS, Canada, 2004

[3] Fernandez J., Material Architecture, emergent materials for innovative buildings and ecological construction, Elsevier, Oxford, 2006

[4] Basti A., 2005, La cultura materiale come innovazione costruttiva, Proc. Of the Int. Conf. On Teoria e pratica del costruire: saperi, strumenti, modelli, eds. G. Mochi, Edizioni Moderna, Ravenna, pp. 1337-1346, 2005

[5] Hendriks F., Durable and sustainable construction materials, Aeneas, Netherlands, 2000 